IDS - Internationale Dental-Schau 2013 in Köln

\section{Tickets ab sofort erhältlich}

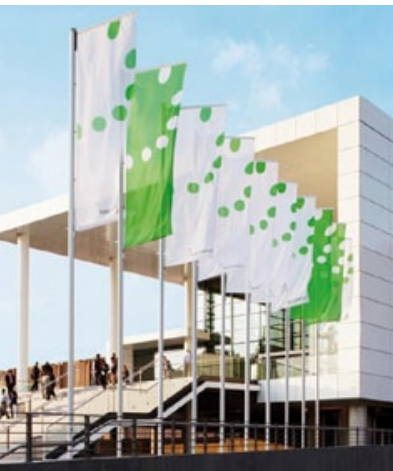

Der Ticket-Shop der Internationalen Dental-Schau ist ab sofort geöffnet. Nach erfolgreicher Registrierung und Bezahlung unter www. idscologne.de können die E-Tickets für die IDS 2013 ganz unkompliziert am eigenen Computer ausgedruckt werden. So vorbereitet gelangen Besucher der IDS vom 12. bis 16. März 2013 auf schnellstem Weg in die Hallen der Koelnmesse. Wer seine Eintrittskarte online im Ticket-Shop kauft, profitiert zudem von günstigeren Preisen und zahlt für die Tageskarte nur 13 Euro statt 16 Euro an der Tageskasse. Auch Eintrittskartengutscheincodes können im Ticket-Shop gegen kostenlose Eintrittskarten umgetauscht werden. Das hat den Vorteil, dass die Eintrittskarte zugleich als Fahrausweis im öffentlichen Personennahverkehr des Verkehrsverbunds RheinSieg (VRS) gilt. Dadurch können die Besucher bereits bei der Anreise kostenlos Busse und Bahnen in Köln und Umgebung nutzen. Zur gezielten Messevorbereitung und zum effizienten Besuch der Veranstaltung tragen zudem diverse digitale Services der Koelnmesse bei. Dazu zählen die eigene IDS-App, das Business-Matchmaking 365, der Online-Terminplaner, der Online-Wegplaner und natürlich die Online-Ausstellersuche, die bereits seit Oktober über die IDS-Website erreichbar ist. Bereits im Vorfeld der Veranstaltung können die Besucher über den Online-Terminplaner einfach per E-Mail Terminanfragen an Aussteller schicken, während der Online-Wegplaner die optimale Route zu den favorisierten Ausstellern durch die Hallen zusammenstellt. Derart gut vorbereitet können sich die Besucher auf der Messe selbst von der eigenen IDS-App für iPhone, Blackberry und weitere Betriebssysteme leiten lassen.

Nach einer Pressemitteilung der

Koelnmesse GmbH, Köln

Internet: www.koelnmesse.de

Unternehmensübernahme

\title{
Stärkung der Position im Bereich Vollkeramik
}

Ivoclar Vivadent mit Hauptsitz in Schaan, Liechtenstein, hat die Übernahme von Wieland Dental bekannt gegeben. Die Akquisition soll im 4. Quartal 2012 abgeschlossen werden. Die Transaktion steht noch unter dem Vorbehalt der Freigabe durch das deutsche Bundeskartellamt. Ivoclar Vivadent erwirbt die Anteile von der Beteiligungsgesellschaft BWK und der Gründerfamilie Wieland. Wieland Dental hat 2011 einen Umsatz von rund 60 Mio. Euro erwirtschaftet und beschäftigt 190 Mitarbeiter.

Ivoclar Vivadent gehört zu den weltweit führenden Dentalunternehmen mit einer umfassenden Produkt- und Systempalette für Zahnärzte und Zahntechniker. Vollkeramik ist eine der Kernkompetenzen des Unternehmens. Mit der Übernahme von Wieland Dental stärkt das Unternehmen seine Position im Bereich der vollkeramischen Produktsysteme. Robert Ganley, CEO des
Unternehmens, zur Übernahme: „Wieland Dental ist ein führender europäischer Hersteller im Dentalbereich mit etablierten Markenprodukten und einem starken Verkaufsteam. Wir freuen uns sehr darüber, dass das Unternehmen zur Ivoclar Vivadent Gruppe stösst.“

Wieland Dental mit Sitz in Pforzheim, gehört zu den führenden Anbietern in der Dentaltechnik. Mit einem breiten Angebotsspektrum, das von CAD/CAM-Materialien über Dentallegierungen und Verblendkeramiken bis zur Galvanotechnik reicht, bietet das Unternehmen individuelle Laborkonzepte und Systemlösungen an. Es soll unter dem bisherigen Namen und Management weitergeführt werden.

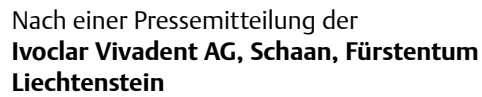

Internet: www.ivoclarvivadent.com

\section{Haftverbund von Zirkonoxid untersucht \\ Forschungspreistråger nehmen Oxidkeramik unter die Lupe}

Seit über einer Dekade berichtet die AG Keramik alljährlich über den Stand der vollkeramischen Restauration und ermuntert junge Wissenschaftler, Technologen, Zahnärzte und Zahntechniker, im Rahmen des „Forschungspreises Vollkeramik“ über die Fortschritte und ihre Erfahrungen zu berichten. Die prämierten Arbeiten und deren Autoren werden alljährlich auf dem Keramiksymposium der AG Keramik vorgestellt. Die eingereichten Arbeiten der 12. Ausschreibung hat die Jury geprüft und die Gewinner des Forschungspreises Vollkeramik ermittelt. Den 1. Preis erhielten PD Dr.-Ing. U. Lohbauer mit der Arbeitsgruppe A. Grigore, S. Spallek, A. Petschelt, B. Butz, E. Spiecker, Institut für Biomaterialien \& Center for Nanoanalysis and Electron Microscopy der Universität Erlangen-Nürnberg sowie die Zahnklinik für Zahnerhaltung und Parodontologie, Werkstoffwissenschaftliches Labor, Universität Erlangen, für die Arbeit „Mikrostrukturelle Untersuchungen an der Grenzfläche zwischen Zirkonoxid und Verblendkeramik“. Der 2. Preis ging an die Zahnärzte P. Winterhalder und C. Holberg, Labor für virtu- elle Biomechanik an der Zahnklinik der Ludwig-Maximilians-Universität München, für die Arbeit „Besteht für grazile Keramik-Inlays ein erhöhtes Frakturrisiko?“

Der 3. Preis wird für gleichwertige Arbeiten geteilt und ging an Dr. U. Beier, Universität Innsbruck, für die Arbeit „Klinische Bewährung vollkeramischer Inlay- und OnlayRestaurationen im Seitenzahnbereich" sowie an Dr. G. Göstemeyer, Charité-Centrum der Humboldt-Universität Berlin, für die Arbeit „Einfluss der Abkühlgeschwindigkeit auf den Haftverbund zwischen Zirkoniumdioxid und Verblendkeramik".

Die Preisträger wurden auf dem 12. Keramiksymposium der AG Keramik auf dem Deutschen Zahnärztetag in Frankfurt/ Main vorgestellt und erhielten ihre Auszeichnungen aus der Hand von Dr. B. Reiss, 1. Vorsitzender der AG Keramik.

Die Zusammenfassungen aller 4 prämierten Arbeiten im Internet unter www.agkeramik.eu, Menü Wissenschaft/Forschungspreis 2012.

Nach einer Pressemitteilung der

AG Keramik, Ettlingen 


\section{Bietigheimer Familienunternehmen gewinnt Wettbewerb der Harvard Clubs of Germany}

Der Deutsche Unternehmer Preis in der Kategorie Nachfolge geht 2012 an die Dürr Dental AG aus Bietigheim-Bissingen. Vorstandsvorsitzender M. Dürrstein und sein Bruder P. Dürrstein nahmen die Auszeichnung am Montagabend, dem 6. November, im Frankfurter Opernturm entgegen. „Als Familienunternehmen in der 3. Generation freuen wir uns über diese Anerkennung für unsere vorbildliche Unternehmensnachfolge“, so Vorstandsvorsitzender Dürrstein. Das Unternehmen hatte sich erstmals an dem Wettbewerb beteiligt, den die Harvard Clubs of Germany seit 2006 alle 2 Jahre ausschreiben. Ziel ist dabei, Vorbilder für mutiges unternehmerisches Handeln zu ehren und zu einer besseren Wahrnehmung von Familienunternehmen in der Öffentlichkeit bei-

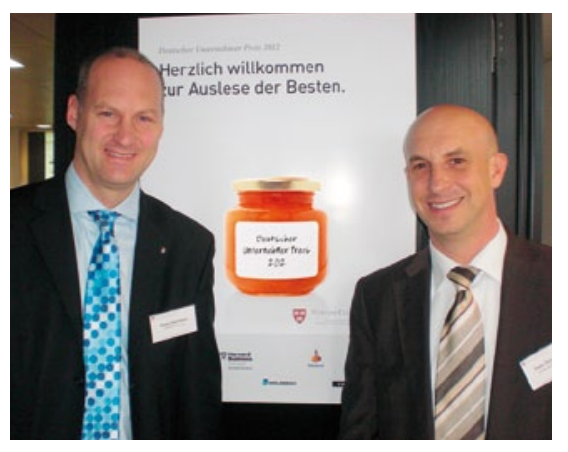

zutragen. Der Deutsche Unternehmer Preis wird in den 4 Kategorien Start-up, Wachstumsunternehmen, Unternehmensnachfolge und Social Entrepreneur verliehen. M. und P. Dürrstein führten im Frankfurter Opernturm aus, wie die Brüder Karl und Wilhelm Dürr aus Gechingen im Schwarzwald 1941 die Dürr
Dental AG als feinmechanische Werkstätte in Stuttgart-Feuerbach gegründet hatten und wie daraus das international agierende Unternehmen wuchs. Heute befindet sich der Firmenhauptsitz in Bietigheim-Bissingen, wo rund 390 Mitarbeiter in den Bereichen Produktion, Forschung und Entwicklung, Einkauf sowie Vertrieb und Marketing beschäftigt sind. Seit 2004 steht die 3. Generation der Familie in der Führungsverantwortung.

Der Preis beinhaltet die Teilnahme an einem Executive Programm der Harvard Business School in Boston. Diese gilt als eine der angesehensten Business Schools der Welt.

Nach einer Presseinformation der DÜRR DENTAL AG, Bietigheim-Bissingen Internet: www.duerr.de 